\title{
GaAs TRIAC-LIKE TRIANGULAR BARRIER SWITCH PREPARED BY MOLECULAR BEAM EPITAXY
}

\author{
M. R. LEF, K. F. YARN*, C. C. CHEN \\ and W. R. CHANG \\ Far East College, Department of Electrical Engineering, Optoelectronic \\ Semiconductor Center, Hsin-Shih, Tainan, Taiwan 744
}

(Received 30 June 2000; In final form 12 September 2000)

\begin{abstract}
A new S-shaped negative differential resistance (NDR) switching device, prepared by molecular beam epitaxy (MBE), has been successfully developed in a GaAs double triangular barrier structure. Symmetrical bidirectional S-shaped NDR characteristics are observed experimentally. The bidirectional current-voltage $(I-V)$ characteristics exhibit a new type of NDR caused by an avalanche multiplication process in reverse biased base-collector region and barrier redistribution. Under a base current injection with respect to the cathode, the device exhibits a conventional transistor with a current gain of 1.2 at room temperature. The experimentally electrical results can be easily understood by an equivalent circuit. In addition, a new optoelectronic switching device is also proposed which may have the potential for bidirectional wave length emission.
\end{abstract}

Keywords: Negative differential resistance; Avalanche multiplication

\section{INTRODUCTION}

Devices with S-shaped negative differential resistance behaviors have been taken a high interest in their potential as functional devices in logic [1], memory [2] and high speed applications [3,4]. In the past few years, a variety of S-shaped NDR devices, including metal-insulatorsemiconductor [5] (MIS) structure and triangular barrier (TB) switch $[6,7]$, double heterostructure optoelectronic switch [8] (DOES) and

*Address for correspondence: P.O. Box 345, Tainan, Taiwan 704, Republic of China. Fax: 886-6-5970454, e-mail: yarn@cc.fec.edu.tw 
heterostructure hot-electron diode [9] $\left(\mathrm{H}^{2} \mathrm{ED}\right)$ etc., have been broadly investigated for their possible applications. Most of them concerned with the I-V characteristics are concentrated on developing the unidirectional switching behavior of heterojunction NDR devices. These devices possess switching behavior only in the forward bias condition, whereas in the reverse bias condition, they function just as a reverse biased diode. However, efforts made for the occurrence of bidirectional bistability switching behavior using the barrier structure are rare and still limited.

Owing to the original work made on the investigations of electrical NDR conduction in a quantum well structure by Esaki et al. $[10,11]$, there has been a growing interest in the development of superlatticed NDR devices. Recently, the AlGaAs/GaAs superlattice structure [12] and sawtooth doping superlattice [13] have been applied to demonstrate the possibly bidirectional $\mathrm{I}-\mathrm{V}$ characteristics using the periodically symmetrical structure in either direction. But, the physical properties of present NDR are strongly dependent on the period length. In addition, a recent report by Darwish et al. [14] using two MIS structures back to back in series, i.e., MISIM structure, has suggested a bidirectional S-shaped NDR. The concept to obtain the expected switching characteristics has been utilized in our study. Thus, a homojunction $\mathrm{GaAs}$ bidirectional bistability switch using $\mathrm{n}^{+}-\mathrm{n}^{-}-$ $\delta\left(\mathrm{p}^{+}\right)-\mathrm{n}^{-}-\mathrm{p}^{+}-\mathrm{n}^{-}-\delta\left(\mathrm{p}^{+}\right)-\mathrm{n}^{-}-\mathrm{n}^{+}$structure, i.e., two isotype regenerative switches back-to-back in series, prepared by MBE is fabricated and characterized. To our knowledge, all thyristors, including unidirectional or bidirectional switching behavior, are regenerative switching devices, and they can not operate in a linear manner like transistors. In this report, we have successfully developed a new switching device which possesses the unidirectional, bidirectional switching performance and bipolar action on the same wafer. The operation mechanism of the bidirectional switch is analyzed by an equivalent circuit. Finally, we also propose an optoelectronic switch, like DOES, such kind of device may have the potential for bidirectional wavelength emission.

\section{EXPERIMENTS}

The studied GaAs bidirectional NDR device was grown by an Anelva 830 S system on a (100)-oriented $n^{+}$-GaAs substrate. The details of 
sample growth and MBE growth procedure have been described elsewhere [15]. The corresponding layer sequence, thickness and doping concentration are listed in Table I. The studied structure, as

TABLE I The grown layer sequence, thickness and doping concentration of the GaAs bidirectional switch

\begin{tabular}{lcc}
\hline Layer & Thickness & Doping $\left(\mathrm{cm}^{-3}\right)$ \\
\hline 1 & $0.4 \mu \mathrm{m}$ & $5 \times 10^{17}$ \\
2 & $100 \mathrm{~A}$ & $5 \times 10^{18}$ \\
3 & $1.0 \mu \mathrm{m}$ & $1 \times 10^{17}$ \\
4 & $0.1 \mu \mathrm{m}$ & $5 \times 10^{18}$ \\
5 & $1.0 \mu \mathrm{m}$ & $1 \times 10^{17}$ \\
6 & $100 \mathrm{~A}$ & $5 \times 10^{18}$ \\
7 & $0.4 \mu \mathrm{m}$ & $5 \times 10^{17}$ \\
8 & $0.2 \mu \mathrm{m}$ & $2 \times 10^{18}$ \\
\hline
\end{tabular}

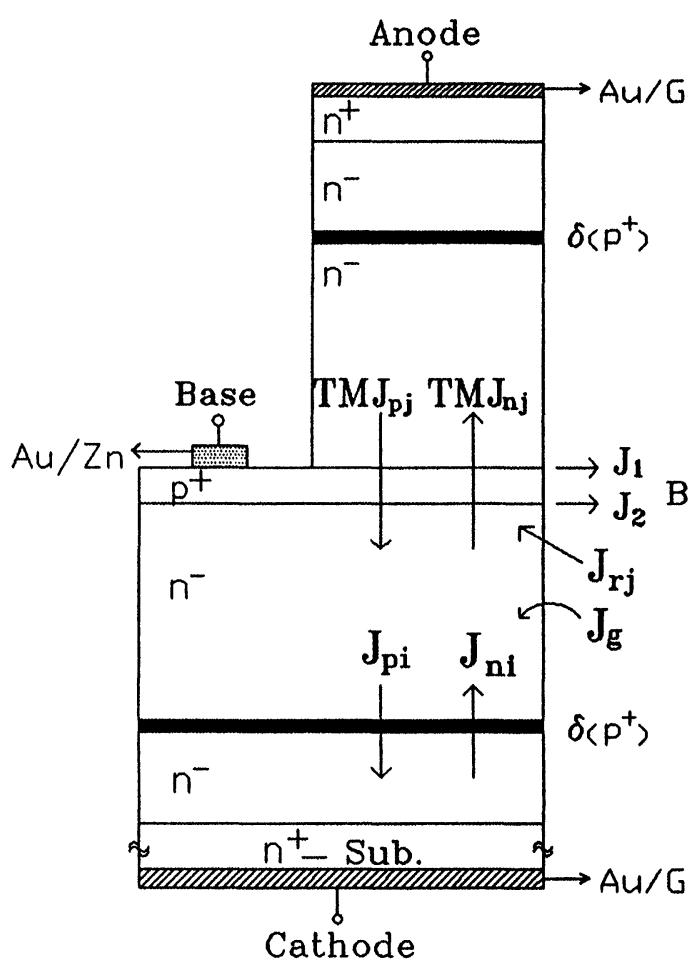

(a)

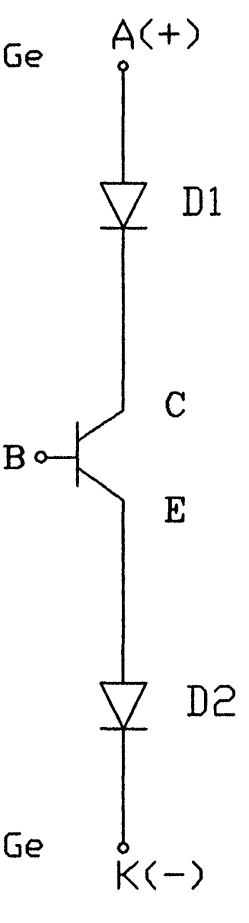

(b)

FIGURE 1 (a) Schematic cross section of the proposed bidirectional switch and relative current components. (b) The equivalent circuit of the studied device. 
illustrated in Figure 1(a), consisted of $0.4 \mu \mathrm{m}$-thick $\mathrm{n}^{-}$layer $\left(5 \times 10^{17} \mathrm{~cm}^{-3}\right)$, followed by a $100 \AA$-thick $\delta\left(\mathrm{p}^{+}\right)=5 \times 10^{18} \mathrm{~cm}^{-3}$ barrier layer, a $1.0 \mu \mathrm{m}$-thick $\mathrm{n}^{-}=1 \times 10^{17} \mathrm{~cm}^{-3}$, a $0.1 \mu \mathrm{m}$-thick base layer $\mathrm{p}^{+}=5 \times 10^{18} \mathrm{~cm}^{-3}$, a $1.0 \mu \mathrm{m}$-thick $\mathrm{n}^{-}=1 \times 10^{17} \mathrm{~cm}^{-3}$, a $100 \AA$-thick $\delta\left(\mathrm{p}^{+}\right)=5 \times 10^{18} \mathrm{~cm}^{-3}$ barrier layer, a $0.4 \mu \mathrm{m}$-thick $\mathrm{n}^{-}=5 \times 10^{17} \mathrm{~cm}^{-3}$, and a $0.2 \mu \mathrm{m}$-thick $\mathrm{n}^{+}=2 \times 10^{18} \mathrm{~cm}^{-3}$ cap layer. The growth temperature was kept at $580^{\circ} \mathrm{C}$ under As-stabilized condition during the MBE growth. After finishing the epitaxial growth, the grown sample was etched in $\mathrm{H}_{2} \mathrm{SO}_{4}: \mathrm{H}_{2} \mathrm{O}_{2}: 8 \mathrm{H}_{2} \mathrm{O}$ solution to form the $100 \times 100 \mu \mathrm{m}^{2}$ mesa islands. $\mathrm{Au} / \mathrm{Zn}$ and $\mathrm{Au} / \mathrm{Ge}$ metals were deposited as ohmic contacts for the p-type base, and the n-type anode, cathode by conventional photolithography and lift-off techniques. The base etching was controlled by the testing pattern to monitor the etching depth in $3 \mathrm{NH}_{4} \mathrm{OH}: \mathrm{H}_{2} \mathrm{O}_{2}: 30 \mathrm{H}_{2} \mathrm{O}$ solution. Finally, all the studied films were measured in the dark using a Tektronix 370A curve tracer and an HP 4145A parameter analyzer at room temperature.

\section{RESULTS AND DISCUSSION}

Basically, this device can be regarded as two GaAs $\mathrm{p}^{+}-\mathrm{n}^{-}-\delta\left(\mathrm{p}^{+}\right)-$ $\mathrm{n}^{-}-\mathrm{n}^{+}$regenerative switches with the $\mathrm{p}^{+}$layer back-to-back connected together. It also can be considered to be the combination of a regenerative switch incorporated with an another $\mathrm{n}^{-}-\delta\left(\mathrm{p}^{+}\right)-\mathrm{n}^{-}-\mathrm{n}^{+}$TB diode. The operation mechanism can be analyzed with the aid of the equivalent circuit shown in Figure 1(b). It consists of two TB diodes, separated by a npn transistor. Two TB diodes are referred to as $D_{1}$ and $D_{2}$, respectively. Due to the bidirectional [16] performance in the TB diode, its $I-V$ characteristics is fully dependent on the barrier height in either direction. If now, an anode voltage is positive biased with respect to the cathode, the junction $J_{2}$ is forward biased, while the junction $J_{1}$ is reverse biased. Most of the applied voltage $\left(\mathrm{V}_{A K}\right)$ will be across $\mathrm{J}_{1}$. At this moment, the internal barriers are sufficiently difficult to overcome by thermionic emission, and introduce the high-impedance off-state. As the applied voltage is increased up to some value, the base-open $\mathrm{CE}$ breakdown voltage $\left(\mathrm{BV}_{C E O}\right)$, the avalanche breakdown will occur in $\mathrm{J}_{1}$ 
( $\mathrm{BC}$ junction). $\mathrm{BV}_{C E O}$ is given by

$$
B V_{C E O}=B V_{C B O}\left(1-\alpha_{o}\right)^{1 / n}
$$

where $\mathrm{BV}_{C B O}$ is the emitter-open avalanche breakdown voltage, $\alpha_{o}$ is the common-base current gain, and $\mathrm{n}$ is a constant. For $\alpha_{o} \sim 1, \mathrm{BV}_{C E O}$ is much smaller than $\mathrm{BV}_{C B O}$. As the hole and electron current densities $\left(\mathrm{MJ}_{p j}\right.$ and $\left.\mathrm{MJ}_{n j}\right)$ greatly increase after avalanche breakdown, $\alpha_{o}$, will increase, causing a reduction in terminal voltage $[17,18]$, where $M$ represents a multiplication factor and defined as $M=1 /\left(1-\left(\mathrm{V}_{C B}\right)\right.$ $\left.\left.\mathrm{BV}_{C B O}\right)^{n}\right)$. Holes, generated by avalanche multiplication, will diffuse toward the base and $\mathrm{D}_{2}$ and accumulate in the potential maxima of the valence band, whereas electrons will diffuse toward $D_{1}$ and accumulate in the potential minima of the conduction band. Owing to screening effect [17], the carrier accumulation in turn lowers the potential barriers, $D_{1}, D_{2}$, leading the potential redistribution in the band structure. Therefore, a positive feedback loop is then established and collapse the internal TB. An switching phenomenon is generated. As to the TB diode, $D_{1}$ it operates just as a diode. The appeared holding voltage is the results of the voltages across $D_{1}, D_{2}$ and the npn transistor. Finally, in steady state, one can get the current components in Figure 1(b) by

$$
\begin{gathered}
J_{n i}+J_{g}=T M J_{n j}+J_{r j} \\
J_{p i}=T M J_{p j}+J_{g} \\
J_{A K}=J_{p i}+J_{n i}
\end{gathered}
$$

where

$\mathbf{J}_{r j}$ : electron recombination current density in junction depletion region.

$\mathrm{J}_{n i}\left(\mathrm{~J}_{p i}\right)$ : electron (hole) injection current density.

$\mathrm{J}_{\mathbf{g}}$ : generation current density.

$\mathrm{T}$ : effective transmission factor.

If the polarity of anode to cathode voltage is reverse now. The bidirectional switch will be turned on by the same mechanism as described above due to the symmetrical structure in either direction. 
In addition, one can obtain the asymmetrical $I-V$ characteristics on purpose with two unequal TB structures.

To investigate $\mathrm{I}-\mathrm{V}$ characteristics of the proposed structure, the observation of unidirectional switching behavior in two regenerative switches of BA and BK are firstly measured. A common principle of these TB switches is that NDR is caused by an increase of hole injection to the potential barrier maximum with an increase in the forward biased pn junction, then, the barrier rapidly collapses, leading to a fast switch from a high-impedance off-state to a low-impedance on-state [19]. Figures 2(a) and 2(b) show the symmetrical I-V characteristics of these two regenerative switches measured at room temperature by a Tektronix 370A curve tracer. The measured switching parameters (i.e., switching voltage, holding voltage, switching current, and holding current) are $\mathrm{V}_{s 1}=4.5 \mathrm{~V}, \mathrm{~V}_{h 1}=2.3 \mathrm{~V}$, $\mathrm{I}_{s 1}=3.8 \mathrm{~mA}, \mathrm{I}_{h 1}=4.8 \mathrm{~mA}$ and $\mathrm{V}_{s 2}=4.5 \mathrm{~V}, \mathrm{~V}_{h 2}=2.3 \mathrm{~V}, \mathrm{I}_{s 2}=3.5 \mathrm{~mA}$, $I_{h 2}=4.4 \mathrm{~mA}$, respectively. Consequently, it is interesting to note that the $I-V$ characteristics between anode $(A)$ and cathode $(K)$ is a significantly symmetrical and bidirectional NDR phenomenon shown in Figure 3. The measured switching parameters in either forward or reverse direction are $\mathrm{V}_{s}^{f}=15.55 \mathrm{~V}, \mathrm{~V}_{h}^{f}=13.7 \mathrm{~V}, \mathrm{I}_{s}^{f}=1.4 \mathrm{~mA}, \mathrm{I}_{h}^{f}=$ $1.7 \mathrm{~mA}$ and $\mathrm{V}_{s}^{r}=-15.6 \mathrm{~V}, \quad \mathrm{~V}_{h}^{r}=-13.8 \mathrm{~V}, \quad \mathrm{I}_{s}^{r}=-1.3 \mathrm{~mA}, \quad \mathrm{I}_{h}^{r}=$ $-1.8 \mathrm{~mA}$ respectively. Two holding voltages are clearly observed as the bidirectional switch is turned on. The calculated electrical field of the reverse biased $B C$ region is greater than $4 \times 10^{5} \mathrm{v} / \mathrm{cm}$ at the switching points $\left(V_{s}^{f}, V_{s}^{r}\right)$. This value is large enough to cause an avalanche multiplication. Thus, the establishment of the regenerative feedback for the collapse of the triangular barriers is predicted as the analyses of the equivalent circuit described above. The minor difference of switching parameters in either direction is due to the variation of doping concentration or device's process. In this device, the voltage difference between $V_{s}$ and $V_{h}$ is small which can be improved by the increase the TB height. The increase of the TB height will result in the increase of $\mathrm{V}_{s}$. The study of device parameters, such as the scaling TBs and the device length for such a new device are under development.

Next, a three-terminal experimental I-V characteristics of the studied switching device under current injection condition is shown in Figure 4. For a positive base current with respect to the cathode, a 
(a)

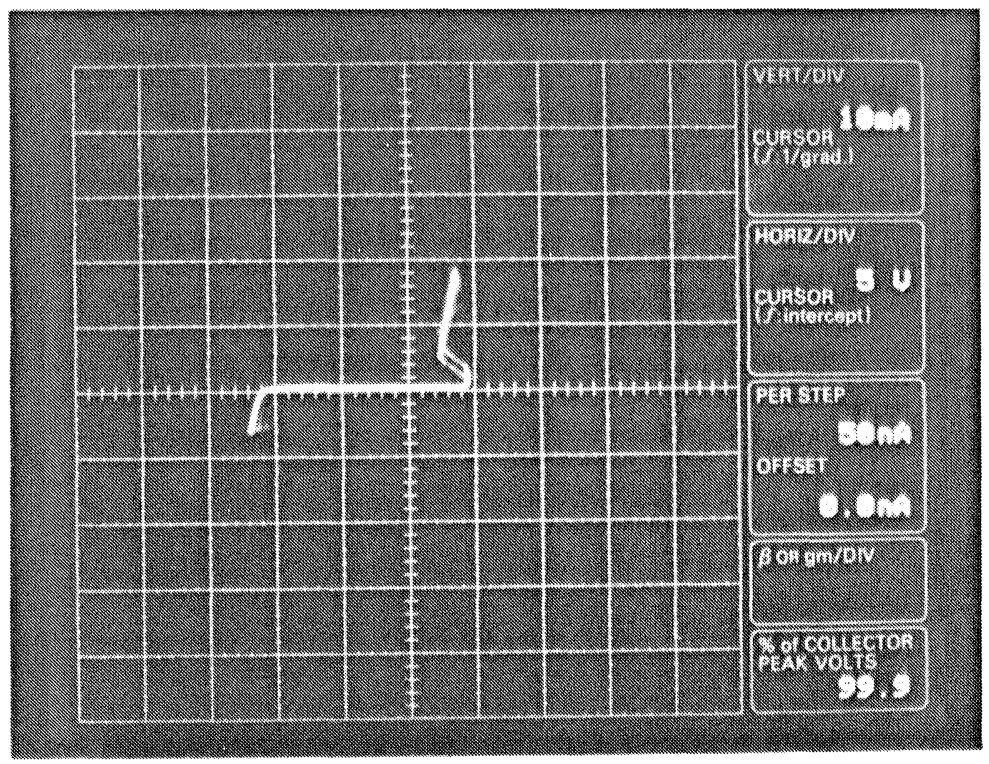

(b)

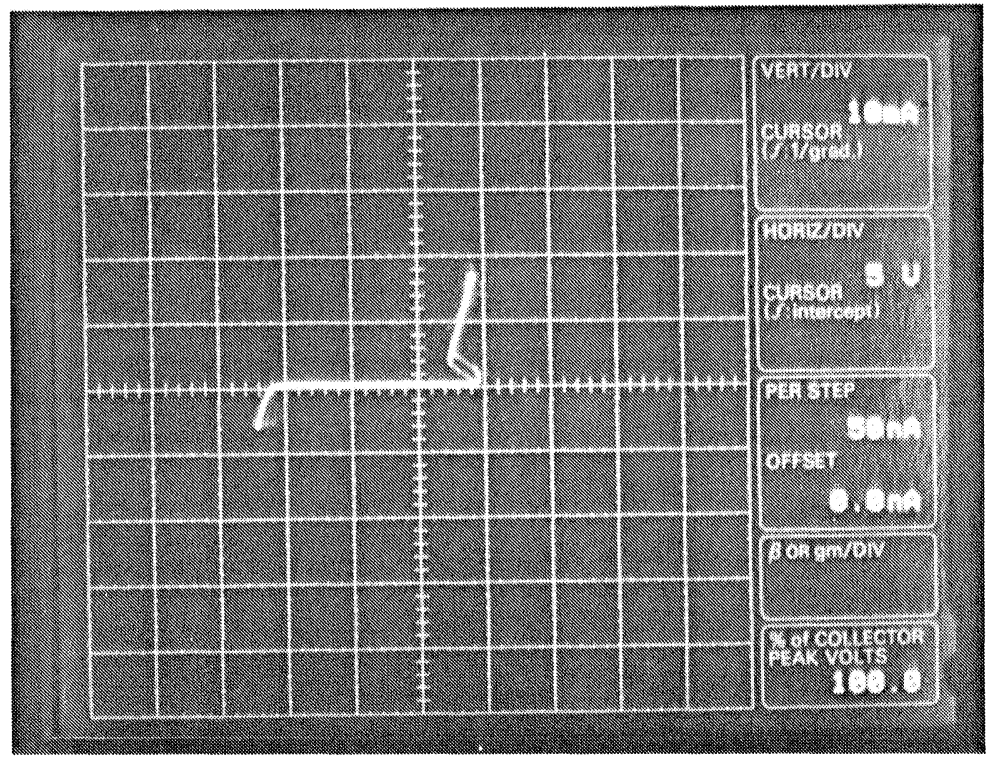

FIGURE 2 I-V characteristics of two regenerative switches at room temperature (a) BA switch (b) BK switch. 


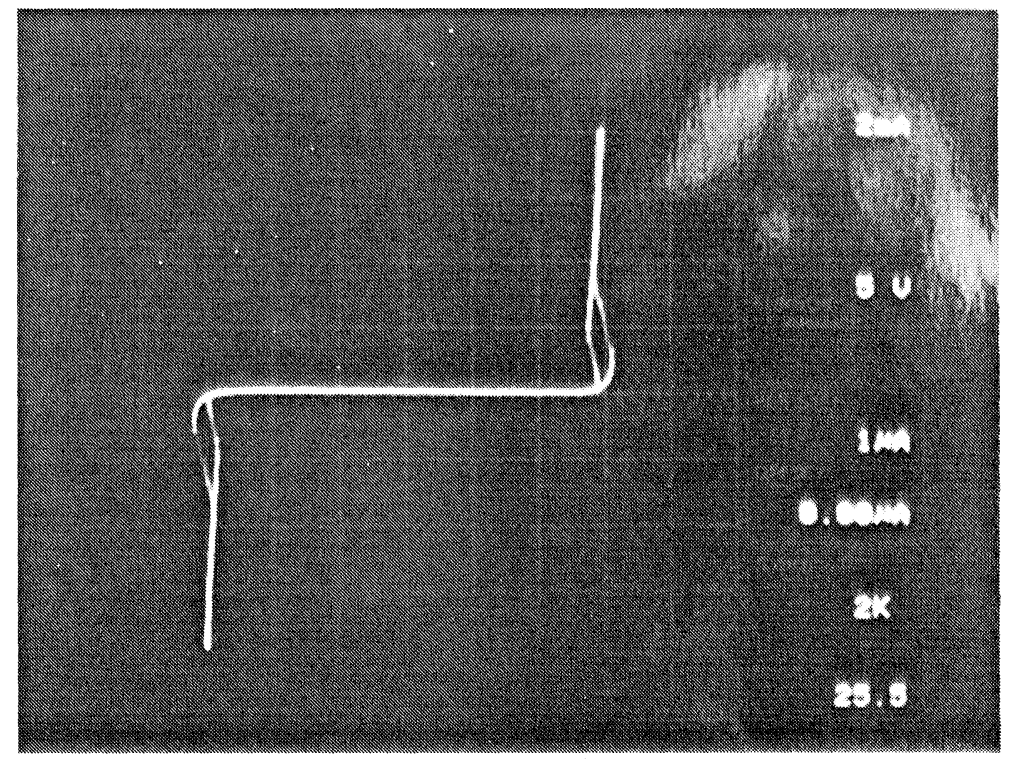

FIGURE 3 I-V characteristics of the proposed bidirectional switch between anode and cathode at room temperature.

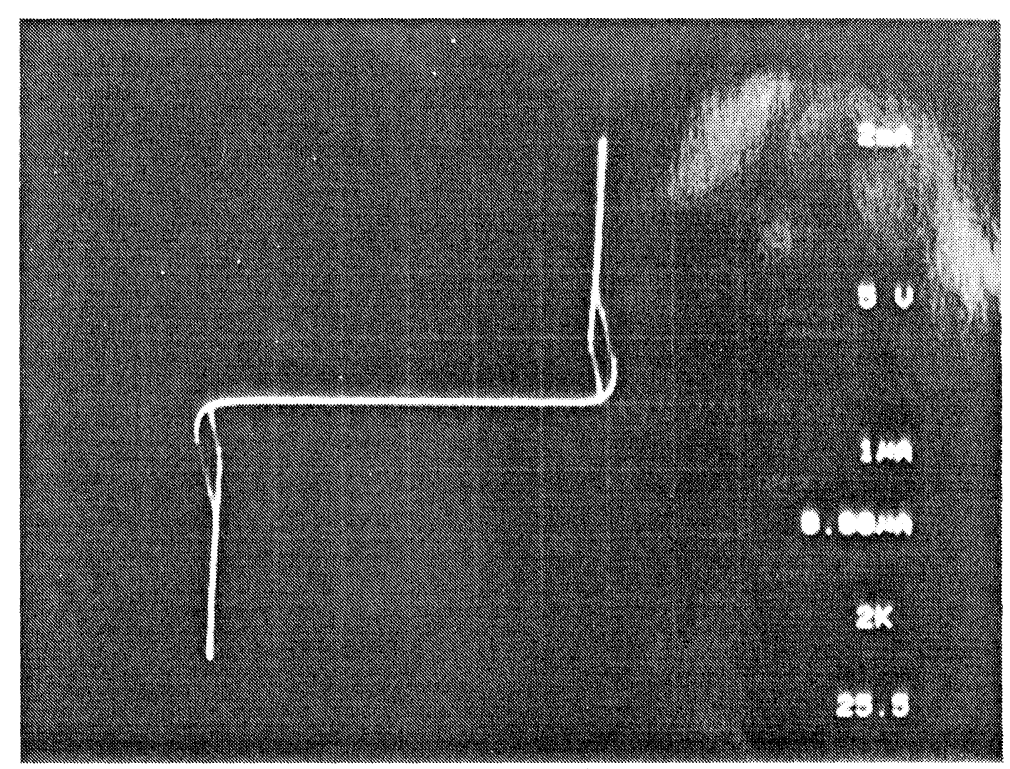

FIGURE 4 Room temperature I-V characteristics of common-emitter configuration under a base current injection with respect to the cathode. 
current gain of 1.2 is obtained. This low value is resulted from the high base doping concentration and wide thickness in collector and emitter regions. In this operation mode, a triac-like device can be also realized

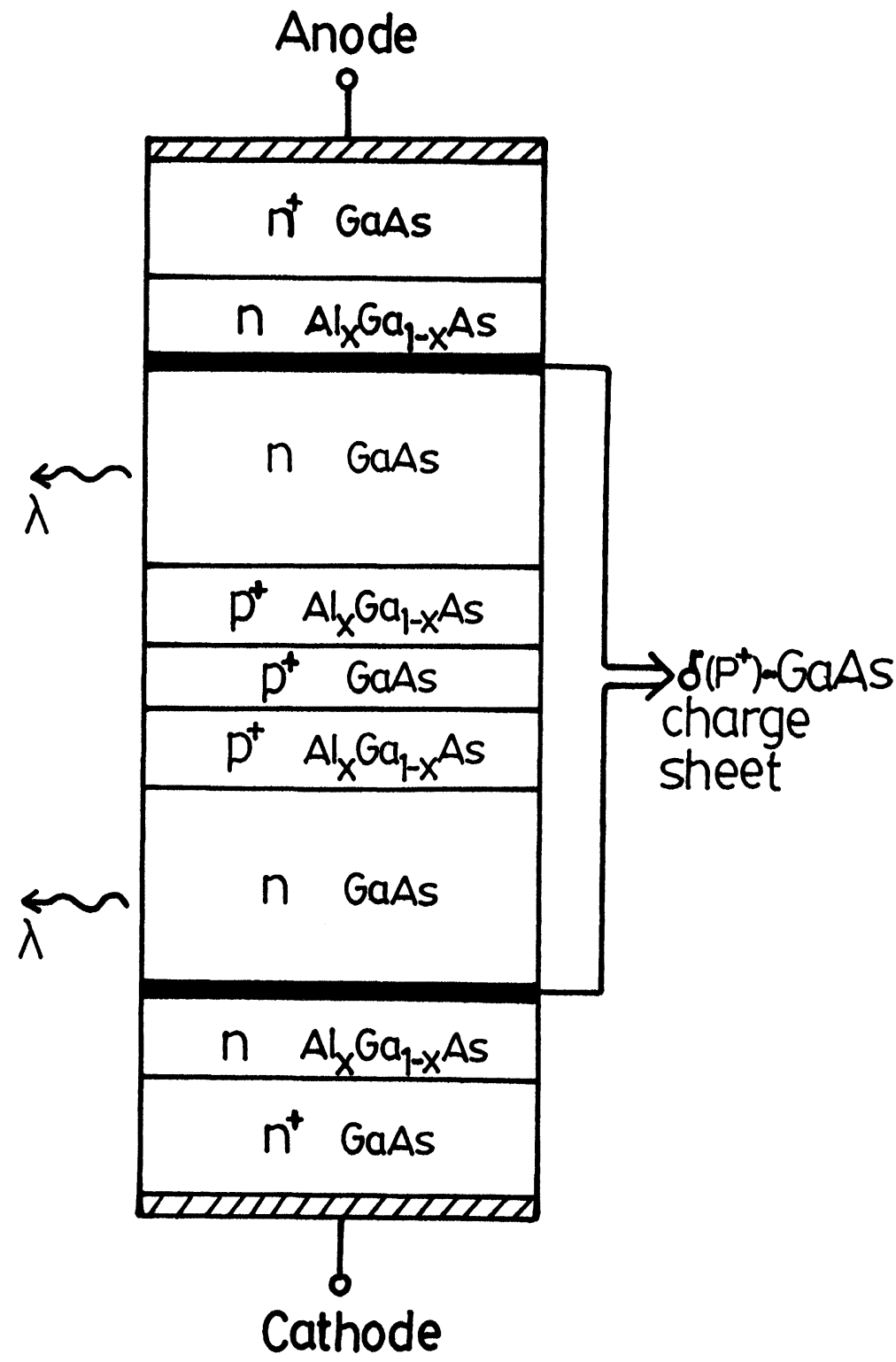

FIGURE 5 The proposed optoelectronic switch for bidirectional wavelength emission. 
by a third electrode contacted to the $\mathrm{n}^{-}$layer of the $\mathrm{npn}$ transistor. This mode is found to be the most effective for the current triggered mode [20]. The detailed results will be published elsewhere. In short, this bidirectional device mainly relies on the $J_{1}$ (BC junction) breakdown to initiate the instability, similar to a diac [17], but it differs in that the majority of the prebreakdown voltage hold off must come from a TB formed by an extremely thin doped epitaxial layer. The existence of different performance operation, i.e., unidirectional, bidirectional switches and bipolar action, on the same wafer may provide more flexibility for device applications. From the bidirectional nature of this device, we also propose a new optoelectronic switching device which is shown in Figure 5. In the off-state, the impedance is high and there is no emission light. In the on-state, the impedance is low and strong emission of light [21] may occur where the on-state conduction is accompanied by the spontaneous emission which is to be expected from the double heterostructures. Thus, compared with other switching devices, this proposed device has the following potential advantages of: (a) the design of symmetrical or asymmetrical I-V characteristics is feasible (b) can operate in either unidirectional or bidirectional switching mode (c) the ability of bidirectional wavelength emission using heterostructure.

\section{CONCLUSIONS}

We have demonstrated the first observation of a unidirection and bidirection combined switching device which possesses a strong and symmetrical bistability phenomenon in a GaAs $\mathrm{n}^{+}-\mathrm{n}^{-}-\delta\left(\mathrm{p}^{+}\right)$ $-\mathrm{n}^{-}-\mathrm{p}^{+}-\mathrm{n}^{-}-\delta\left(\mathrm{p}^{+}\right)-\mathrm{n}^{-}-\mathrm{n}^{+}$structure. A bidirectional switching behavior, which is attributed to the avalanche multiplication process occurring within $\mathrm{BC}$ junction and the $\mathrm{TB}$ redistribution, is obvious in either direction of experimental $\mathrm{I}-\mathrm{V}$ characteristics. In three-terminal operation, a conventional transistor behavior and switching phenomenon is obtained, simultaneously. The best value of current gain is 1.2. Owing to the reproducible switching characteristics and three-terminal operation capability, an optimized design on device performance will bring it to have great potential for optoelectronic applications in future. 


\section{References}

[1] Kawai, S., Tashiro, Y., Ichinose, H., Kasahara, K. and Kubota, K. (1989). Opt. Comput. Tech. Dig., 9, 193.

[2] Kasahara, K., Tashiro, Y., Hamao, N., Sugimoto, M. and Yanase, T. (1988). Appl. Phys. Lett., 52, 679.

[3] Taylor, G. W., Mand, R. S., Simmons, J. G. and Cho, A. Y. (1986). Appl. Phys. Lett., 49, 1406.

[4] Ree, P. K. and Barnard, J. A. (1985). IEEE Trans. Electron Devices, ED-32, 1741

[5] Yamamoto, T. and Morimoto, M. (1972). Appl. Phys. Lett., 20, 269.

[6] Habib, S. E. D. and Board, K. (1983). IEE Proc. pt. I, 130, 292.

[7] Board, K. and Darwish, M. (1982). Solid-State Electron, 25, 571.

[8] Simmons, J. G. and Mand, R. S. (1991). Asia-Pacific Engineering Journal, 1, 59.

[9] Hess, K., Higman, T. K., Emanuel, M. A. and Coleman, J. J. (1986). J. Appl. Phys., 60, 3775 .

[10] Esaki, L. (1958). Phys. Rev., 109, 603.

[11] Esaki, L. and Tsu, R. (1970). IBM J. Rev. Dev., 14, 61.

[12] Alferor, Zh. I., Mezrin, O. A., Sinitsyn, M. A., Troshkor, S. I. and Yavich, B. S. (1987). Sov. Phys. Semi-cond., 21, 304.

[13] Schubert, E. F., Cunningham, J. E. and Tsang, W. T. (1987). Appl. Phys. Lett., 51, 817.

[14] Darwish, M. and Board, K. (1981). IEE Proc., pt. I, 128, 161.

[15] Wang, Y. H., Liu, W. C., Chang, C. Y. and Liao, S. A. (1986). J. Vac. Sci. Technol., B4(1), 30.

[16] Malik, R. J., Aucoin, T. R., Ross, R. L., Board, K., Wood, C. E. C. and Eastman, L. F. (1980). Electron. Lett., 16, 836.

[17] Sze, S. M. (1981). Physics of Semiconductor Devices, 2nd edn., Wiley Ch. 1-4.

[18] Shen, G. D., Xu, D. X., Millander, M., Hansson, G. V. and Svensson, C. (1988). IEEE Electron Device Lett., 9, 453.

[19] Wang, Y. H., Yarn, K. F. and Chang, C. Y. (1990). J. Appl. Phys., 29, L243.

[20] Wang, Y. H., Yarn, K. F. and Chang, C. Y. (1990). Int. J. Electron, 68, 693.

[21] Taylor, G. W., Mand, R. S., Simmons, J. G. and Cho, A. Y. (1987). Appl. Phys. Lett., 50, 338. 

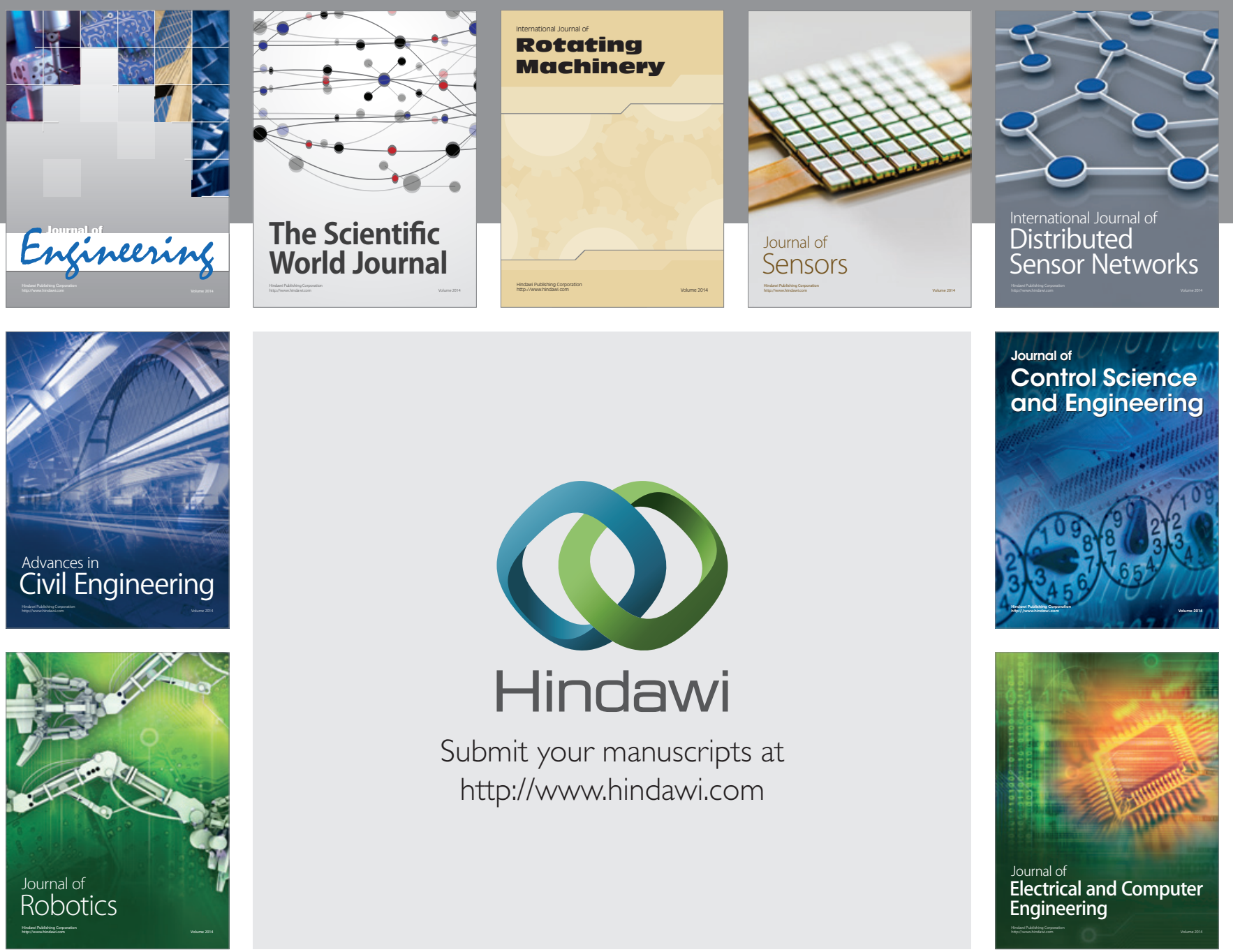

Submit your manuscripts at

http://www.hindawi.com
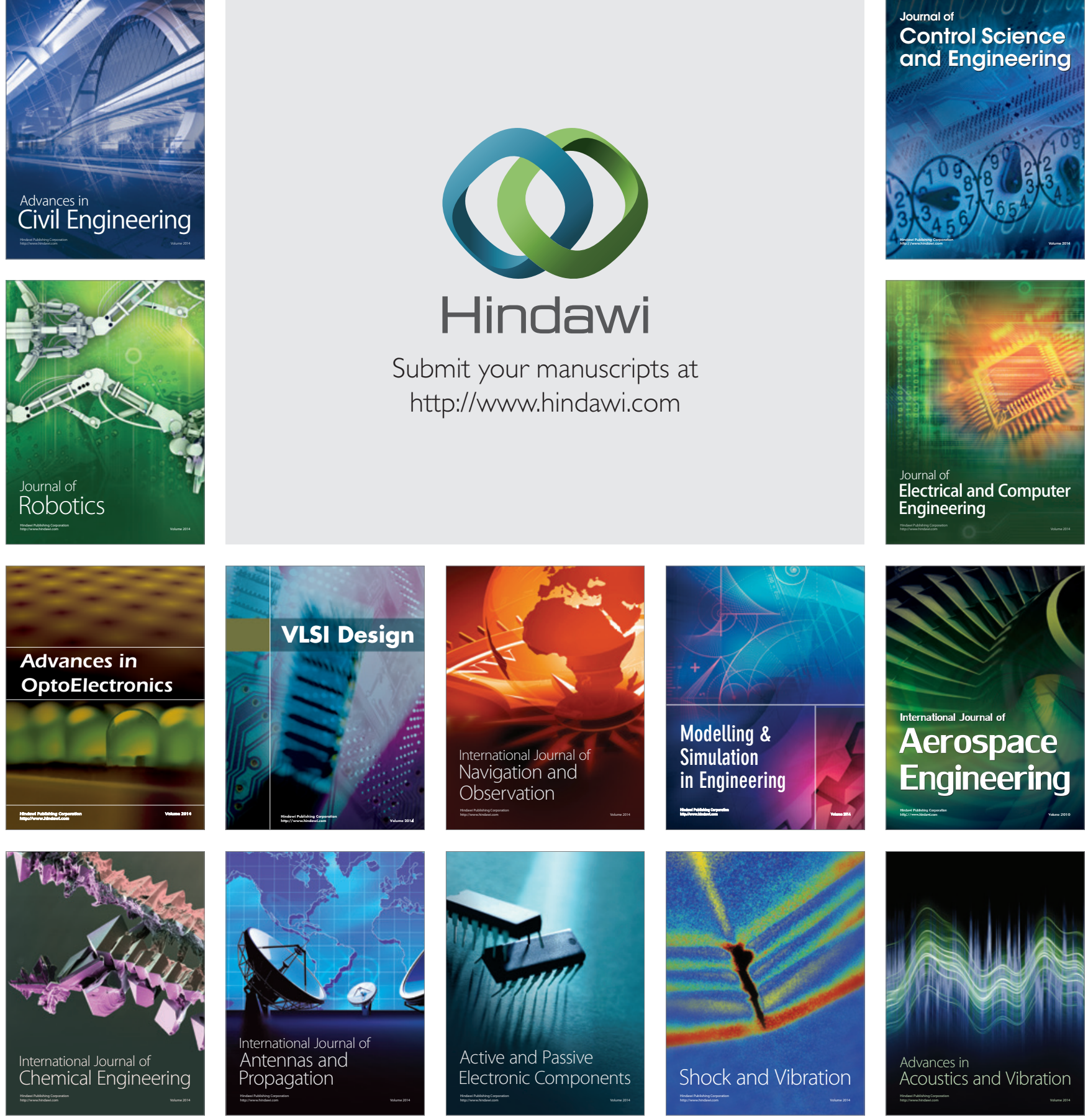